\title{
Comparison of average estimated metabolic rates for styrene in previously exposed and unexposed groups with pharmacokinetic modelling
}

\author{
Yue Wang, Lawrence L Kupper, Agneta Löf, Stephen M Rappaport
}

\begin{abstract}
Objective-To understand whether previous styrene exposure increases the human liver's ability to convert styrene into styrene oxide.

Methods-The hypothesis was tested that the average linear metabolic rate constant $\kappa$ was the same in both exposed and unexposed groups, when the exposed group comprised people with a history of styrene exposure and the unexposed group had no exposure. In an experimental chamber, these two groups of subjects were exposed to a concentration of 80 ppm styrene for two hours. A three compartment pharmacokinetic model was used to define $\kappa$. Based on large sample theory, the comparison of estimated mean values of $\kappa$ in the exposed and unexposed groups was shown to be equivalent to a comparison of the estimated mean values of the hepatic clearance $X$ in the two groups. A method was developed to estimate $\mathbf{X}$ for each subject in both groups from the subject's height, weight, and estimated asymptotic styrene decay constant $\alpha$. Here, $\alpha$ was estimated individually from observed blood concentrations over time when sufficient time had elapsed after the controlled exposure.
\end{abstract}

Results-The proposed methodology of comparing the estimated mean values of $\kappa$ in exposed and unexposed groups reduced the number of specific physiological variables involved to three, all of which were estimable from data based on simple direct measurements. In contrast, other methods based on pharmacokinetic models usually involved many variables that were non-estimable on an individual basis. Consequently, statistical comparisons were impossible. These methods were applied to analyse previously published data on the time course of styrene concentrations in arterial blood of subjects in both exposed and unexposed groups. A Wilcoxon non-parametric rank sum test with the individually estimated $X$ values was used, and no significant difference in the means of $X$ in the two groups was found.

Conclusion-The linear metabolic rate constant $\kappa$ for humans is probably not altered by previous exposure to styrene. This result is in agreement with some experimental studies on animals. However, in the data analysis, it was noted that the number of subjects in each group was small (6-7) and that the styrene concentration data did not exactly reflect true behaviour of asymptotic decay. Further studies are still needed to draw more definitive conclusions.

(Occup Environ Med 1996;53:601-605)

Keywords: styrene; enzyme induction; pharmacokinetic model; metabolic rate

Most genotoxic effects of styrene are likely to be due to styrene-7,8-oxide (SO), ${ }^{1}$ which is a labile intermediate metabolite of styrene activated by microsomal enzymes. ${ }^{2}$ This is subsequently detoxified into styrene glycol, ${ }^{2}$ which is then oxidised to mandelic acid and phenylglyoxylic acid, ${ }^{3}$ both of which are excreted in urine. There is speculation that previous exposure to styrene may induce CP450 enzymes and thereby increase the biotransformation of styrene into SO. Andersen $e t \mathrm{al}^{4}$ found that the blood styrene concentrations were lower in rats that had been previously exposed to styrene. From this they reasonably concluded that styrene induced its own metabolism. Elovaara et $a^{5}$ showed that styrene metabolism in vitro by liver microsomes was greatly increased after a period of exposure to styrene $\left(2100 \mathrm{mg} / \mathrm{m}^{3}\right.$ for 24 hours) in rats. On the other hand, Mendrala et $a l^{6}$ found that monooxygenase activity was not changed in liver microsomes of rats and mice that had been exposed for six hours a day for three days to $600 \mathrm{ppm}$ styrene. Likewise, Filser $e \mathrm{al}^{7}$ exposed rats and mice to 150 and $500 \mathrm{ppm}$ styrene on five consecutive days (6 $\mathrm{h} /$ day) and found no change in the rate of styrene metabolism compared with non-pretreated controls.

Unlike in animal studies, direct measurements of metabolic activity are difficult to make in humans. This paper develops the theoretical framework for testing the null hypothesis that the average level of metabolic activity is the same in groups with and without previous exposure to styrene. Then the methods are evaluated with data from Wigaeus et al and Löf et al, ${ }^{89}$ who exposed groups of people with and without previous exposure to styrene at $80 \mathrm{ppm}$ for two hours in an exposure chamber. Arterial concentrations of styrene were measured both during and after exposure. To measure the level of metabolic activity, we present a three compartment pharmacokinetic model to simulate the uptake, distribution, metabolism, and elimination of styrene and to define the linear metabolic rate constant $\kappa$. We show that the estimated 
mean value of $\boldsymbol{\kappa}$ in each group is proportional to the estimated mean value of the hepatic clearance $\mathrm{X}$ (defined in equation (6)) in that group. When the number of subjects in each group is large, the proportionality constants for the two groups become close to one another. Consequently, for large samples, we can test our null hypothesis through a comparison of the estimated mean values of $X$.

Normally, to estimate $\kappa$ and other variables, the use of a physiologically based pharmacokinetic model requires detailed information, such as tissue blood flow rate, cardiac output, tissue volume, ventilation, styrene concentration in blood over time, etc. Unfortunately, most of these input variables are not directly measurable and are replaced by some standard values that are not specific to each person (see table 3 ). As a result, variables like $\kappa$ are generally not uniquely estimated, and hence the precision of such estimates is unknown. Our proposed methodology avoids many of these drawbacks. We derive a simple estimator for $\mathrm{X}$ from the asymptotic behaviour of the styrene concentration in blood after exposure. This estimator involves only three estimable physiological variables specific to each person, namely, height, weight, and the asymptotic decay constant $\alpha$, where $\alpha$ is estimated from the decay of styrene concentrations in blood after the end of exposure.

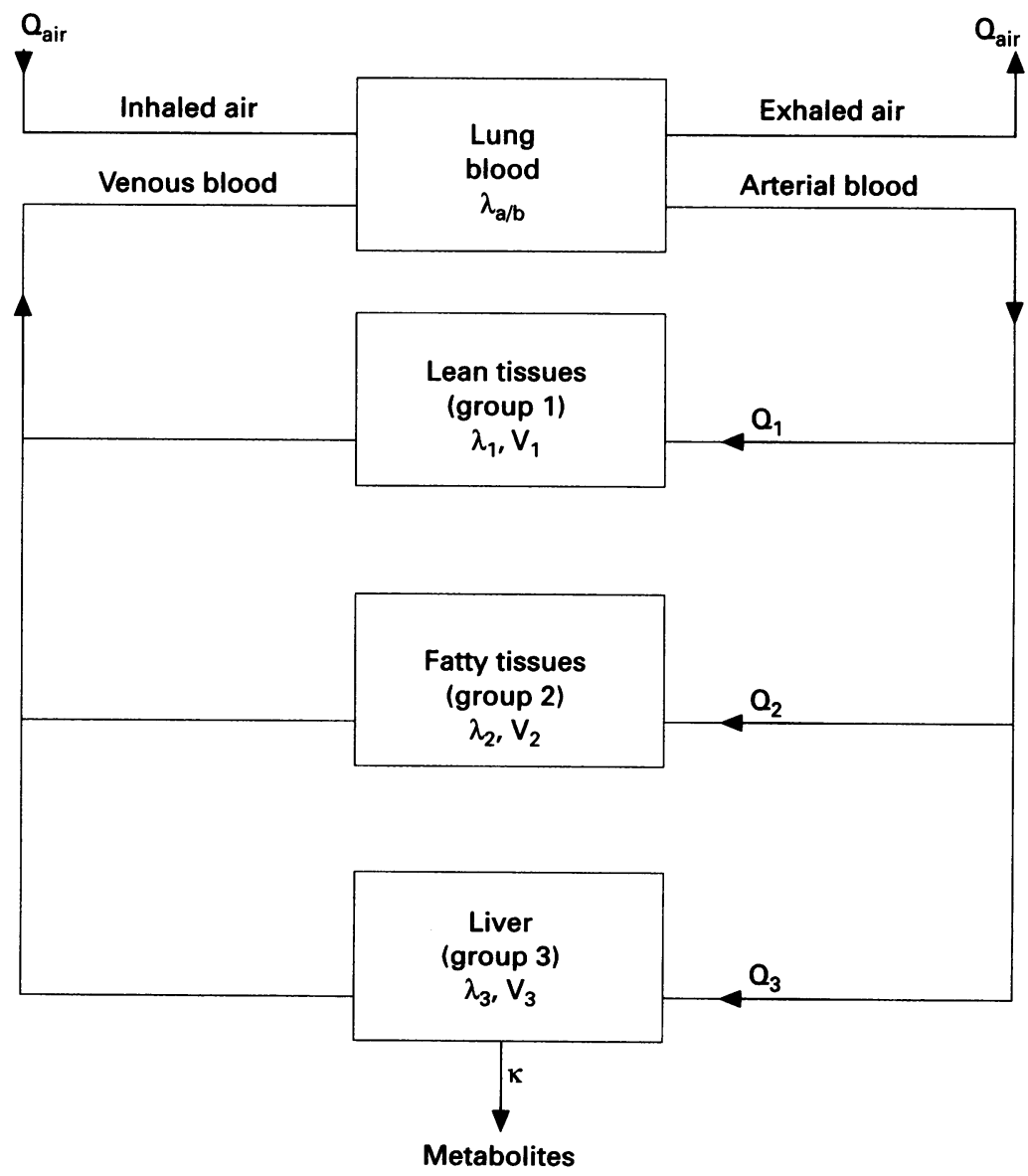

Physiologically based three compartment model. Under moderate physical exercise, the body tissues can be grouped into well perfused tissues (lean tissues), poorly perfused tissues (fatty tissues), and the liver (the main metabolising organ). The time dependent behaviour of styrene concentration in blood is determined by a set of differential equations based on mass conservation. These equations involve the physiological variables shown here.

\section{Methods}

DESCRIPTION OF THE MODEL

We assume that the metabolism of styrene takes place exclusively in the liver, although some studies suggest that pulmonary tissues and bone marrow may also participate to a small degree. ${ }^{10}$ Under moderate physical exercise, the skin and muscle tissues become well perfused, as do blood vessel rich tissues like the brain, kidney, and viscera. Therefore, the body tissues can be roughly grouped into lean tissues $(i=1)$, fatty tissues $(i=2)$, and the liver $(i=3)$ (figure). We assume that each type of tissue has similar composition - that is, the percentages of lipid and water-and similar blood perfusion, and can, therefore, be characterised by a single tissue/blood partition coefficient $\lambda_{i}$ and a single blood flow rate $Q_{i}$ (in $1 / \mathrm{min}$ ). Throughout this paper, unless otherwise specified, time is in minutes, volume in litres, and concentration in $\mathrm{mg} / \mathrm{l}$.

For $\mathrm{i}=1$ and 2 , we have a simple mass balance equation:

$$
\frac{d C_{i}(t)}{d t}=\frac{Q_{i}}{V_{i}}\left[C_{a n}(t)-\frac{C_{i}(t)}{\lambda_{i}}\right]
$$

where $C_{i}(t)$ and $C_{\text {arr }}(t)$ are, respectively, the styrene concentration in the $i$-th tissue group and in the arterial blood, and where $V_{i}$ is the effective volume of the $i$-th tissue group. For the liver, we have an additional term to represent the metabolism of styrene:

$$
\frac{d C_{3}(t)}{d t}=\frac{Q_{i}}{V_{i}}\left[C_{a r}(t)-\frac{C_{3}(t)}{\lambda_{3}}\right]-\kappa C_{3}(t)
$$

where the metabolic rate has been assumed to be first order, as characterised by the constant $\kappa\left(\mathrm{min}^{-1}\right)$.

\section{ASYMPTOTIC VARIABLES}

During the period after exposure, the blood concentration is found to decline in two phases: a first distribution phase, in which concentrations of styrene in all tissues except the fat come into rough equilibrium with the arterial blood concentration, and a second phase, in which the primary mechanism of the decay is metabolism. Once equilibrium throughout the body tissues has been more or less established, the whole body can be treated as a single compartment. ${ }^{11}$ Then the equation of conservation of mass gives:

$$
\sum_{i=1}^{3} \frac{d C_{a r}(t)}{d t} V_{i} \lambda_{i}=-\lambda_{a / b} C_{a r}(t) Q_{a i r}-\kappa V_{3} \lambda_{3} C_{a r t}(t)
$$

where $Q_{\text {air }}$ denotes the alveolar ventilation and $\lambda_{a / b}$ the air/blood partition coefficient.

We solve this equation for $C_{a r t}(t)$ to obtain:

$$
C_{a n}(t)=C_{0} \exp (-\alpha t)
$$

where $C_{0}$ is a constant independent of $t$, and where:

$$
\alpha=\frac{\lambda_{a / b} Q_{a i r}+\kappa V_{3} \lambda_{3}}{\sum_{i=1}^{3} V_{i} \lambda_{i}}
$$

is the decay constant for styrene concentration in blood. We can estimate $\alpha$ by fitting 
Table 1 Styrene concentration ( $\mu$ molll) in arterial blood at various times (min) after 2-hour exposure to 80 ppm styrene for groups with and without earlier exposure to styrene (heights and weights of all subjects are presented)

\begin{tabular}{|c|c|c|c|c|c|c|c|c|c|c|c|c|c|c|c|c|}
\hline \multirow[b]{2}{*}{ Time } & \multicolumn{8}{|c|}{ Group without earlier exposure to styrene } & \multicolumn{8}{|c|}{ Group with earlier exposure to styrene } \\
\hline & 1 & 2 & 3 & 4 & 5 & 6 & 7 & 8 & 1 & 2 & 3 & 4 & 5 & 6 & 7 & 8 \\
\hline $\begin{array}{l}75 \\
90 \\
105 \\
120 \\
180 \\
240 \\
\text { Height (m) } \\
\text { Weight (kg) }\end{array}$ & $\begin{array}{l}1 \cdot 45 \\
1 \cdot 1 \\
1 \cdot 16 \\
0 \cdot 45 \\
0 \cdot 35 \\
0 \cdot 54 \\
180 \\
80\end{array}$ & $\begin{array}{l}1 \cdot 29 \\
0 \cdot 82 \\
1 \cdot 01 \\
0.53 \\
0.51 \\
0 \cdot 38 \\
178 \\
68\end{array}$ & $\begin{array}{l}- \\
- \\
\overline{-} \\
\overline{180} \\
70\end{array}$ & $\begin{array}{l}2 \cdot 97 \\
3 \cdot 28 \\
2 \cdot 44 \\
1 \cdot 42 \\
1 \cdot 2 \\
174 \\
72\end{array}$ & $\begin{array}{c}3.91 \\
2 \cdot 15 \\
1.69 \\
1.35 \\
1.52 \\
1.04 \\
178 \\
60\end{array}$ & $\begin{array}{l}2 \cdot 85 \\
2 \cdot 65 \\
2 \cdot 02 \\
1 \cdot 45 \\
1 \cdot 49 \\
0 \cdot 82 \\
191 \\
79\end{array}$ & $\begin{array}{l}2 \cdot 86 \\
2 \cdot 16 \\
-1 \cdot 4 \\
1 \\
0 \cdot 98 \\
172 \\
68\end{array}$ & $\begin{array}{c}1.94 \\
1.36 \\
2 \cdot 09 \\
1.76 \\
2 \cdot 41 \\
1.07 \\
179 \\
67\end{array}$ & $\begin{array}{l}1 \cdot 17 \\
1 \cdot 14 \\
1 \cdot 38 \\
0 \cdot 77 \\
= \\
\overline{182} \\
69\end{array}$ & $\begin{array}{l}2 \cdot 2 \\
1 \cdot 91 \\
1 \cdot 78 \\
\overline{-} \\
174 \\
72\end{array}$ & $\begin{array}{l}1.91 \\
2 \cdot 34 \\
1.23 \\
1.25 \\
= \\
\overline{188} \\
105\end{array}$ & $\begin{array}{l}2 \cdot 0 \\
2 \cdot 13 \\
1 \cdot 27 \\
1 \cdot 09 \\
= \\
\overline{173} \\
74\end{array}$ & $\begin{array}{l}3 \cdot 92 \\
2 \cdot 6 \\
2 \cdot 38 \\
1 \cdot 56 \\
= \\
\overline{176} \\
72\end{array}$ & $\begin{array}{c}3.33 \\
1.92 \\
1.92 \\
1.29 \\
-\quad \\
\overline{170} \\
69\end{array}$ & $\begin{array}{c}1.44 \\
1.39 \\
0.71 \\
0.9 \\
- \\
\overline{180} \\
68\end{array}$ & $\begin{array}{c}1 \cdot 54 \\
1 \cdot 38 \\
1 \cdot 33 \\
1 \cdot 35 \\
- \\
- \\
179 \\
78\end{array}$ \\
\hline
\end{tabular}

equation (4) to the final portion of the decay curve with least squares method. Note that the styrene concentration in arterial blood should be equal to that in the venous blood after equilibrium is reached. With this estimated $\alpha$ we can then estimate the hepatic clearance ( $1 / \mathrm{min})$ :

$$
\mathrm{X}=\kappa V_{3} \lambda_{3}
$$

as

$$
\hat{\mathrm{X}}=\hat{\alpha} \sum_{i=1}^{3} \hat{V}_{i} \lambda_{i}-\lambda_{a / b} \hat{Q}_{\text {air }}
$$

(note that the hepatic clearance defined in (6) is not the "apparent clearance" defined in Wigaeus et $\left.a^{8}\right)$. As the partition coefficient $\lambda_{2}$ (adipose tissue/ blood) is about 50 times as large as $\lambda_{1}$ (lean tissue/blood) and is over 4000 times as large as $\lambda_{a b}$ (see table 3$)^{3}$, the dominant contribution in equation (7) comes from the adipose tissues. Hence:

$$
\hat{\mathrm{X}} \approx \hat{\alpha} \hat{V}_{2} \lambda_{2}
$$

\section{STATISTICAL COMPARISON}

Suppose we have a random sample of subjects of size $n_{j}$ from the $j$-th group, where $j=1$ pertains to the group with previous exposure to styrene and $j=2$ to the previously unexposed group. All physiological variables vary from person to person. In a sense, each such variable can be considered to have a distribution determined by its varying values across a population of subjects. For subject $k\left(k=1, \ldots, n_{j} ; j\right.$ $=1,2)$, we can estimate the hepatic clearance $\mathrm{X}_{\mathrm{k}}(\mathrm{j})$ according to equation (8). The unbiased estimators of the mean and variance of the underlying distribution of hepatic clearance values for the $j$-th population are:

Table 2 Estimates of the decay constant $\hat{\alpha}\left(\min ^{-1}\right)$, adipose tissue volume $\hat{V}_{2}(\mathcal{l})$, and the

\begin{tabular}{|c|c|c|c|c|c|c|}
\hline \multirow[b]{2}{*}{ Subject } & \multicolumn{3}{|c|}{ Group with earlier exposure } & \multicolumn{3}{|c|}{ Group without earlier exposure } \\
\hline & $\hat{V}_{2}$ & $\hat{a}$ & $\hat{\boldsymbol{X}}$ & $\hat{V}_{2}$ & $\hat{\alpha}$ & $\hat{\boldsymbol{X}}$ \\
\hline $\begin{array}{l}1 \\
2 \\
3 \\
4 \\
5 \\
6 \\
7 \\
8 \\
\text { Mean }\end{array}$ & $\begin{array}{c}8 \cdot 4 \\
17 \cdot 5 \\
52 \cdot 0^{\star} \\
20 \cdot 8 \\
16 \cdot 2 \\
16 \cdot 1 \\
8 \cdot 4 \\
22 \cdot 2 \\
15 \cdot 7 \dagger\end{array}$ & $\begin{array}{l}0.0053 \\
0.0046 \\
0.0116 \\
0.0132 \\
0.0193 \\
0.0208 \\
0.0142 \\
0.003 \\
0.0115 t\end{array}$ & $\begin{array}{c}3 \cdot 1 \\
5 \cdot 6 \\
42 \cdot 8 \\
19 \cdot 4 \\
22 \cdot 2 \\
23 \cdot 7 \\
8 \cdot 4 \\
4 \cdot 7 \\
12 \cdot 7 \dagger\end{array}$ & $\begin{array}{c}24 \cdot 2 \\
9 \cdot 7 \\
11 \cdot 0 \\
17 \cdot 5 \\
-0.99^{\star} \\
15 \cdot 7 \\
13.5 \\
7 \cdot 7 \\
14.7 \ddagger\end{array}$ & $\begin{array}{l}0.0072 \\
0.0068 \\
0.0067 \\
0.0025 \\
0.0051 \\
0.0033 \\
0.0027 \\
0.0053 \ddagger\end{array}$ & $\begin{array}{c}12 \cdot 3 \\
4 \cdot 6 \\
- \\
8 \cdot 2 \\
- \\
5 \cdot 6 \\
3 \cdot 1 \\
1 \cdot 4 \\
5 \cdot 5 \ddagger\end{array}$ \\
\hline
\end{tabular}
hepatic clearance $\hat{X}(\mathrm{l} / \mathrm{min})$ of all subjects in groups with and without earlier exposure to styrene

*The estimate may not be valid because the subject's height or weight are outside the range over which the regression model (12) is constructed. which the regression mode

†Subject 3 is excluded. 3 and 5 are excluded.

$$
\begin{gathered}
\hat{\mathrm{E}}(\mathrm{X}(\jmath))=\frac{1}{n_{j}} \sum_{k=1}^{n_{j}} \hat{\mathrm{X}}_{k}(j) \\
\left.\hat{V}(\mathrm{X}(j))=\frac{1}{\left(n_{j}-1\right)} \sum_{k=1}^{n_{j}}\left[\hat{\mathrm{X}}_{k}(\jmath)-\hat{E}(\mathrm{X}(\jmath))\right)\right]^{2}
\end{gathered}
$$

where $j=1,2$.

As the linear metabolic rate constant $\kappa$ measures the proportion of the amount of styrene in a unit volume of liver that can be converted to SO in a unit of time, it is therefore independent of the liver's volume $V_{3}$. From equation (6), we obtain:

$$
\hat{E}(\kappa(j))=\frac{\hat{E}(\mathrm{X}(j))}{\lambda_{3} E\left(V_{3}(j)\right)}
$$

where $j=1,2$, and where it is assumed that $\kappa$ and $V_{3}$ are independent for each subject. If $n_{i}$ $(j=1,2)$ is large, we expect that both $E\left(V_{3}(1)\right)$ and $E\left(V_{3}(2)\right)$ would approach the common population mean $\mathrm{E}\left(\mathrm{V}_{3}\right)$. Consequently, a comparison of the means of the metabolic rate constants in the two groups can be made through a comparison of the corresponding means of the estimated hepatic clearances. When sample sizes are small, the above statement is still approximately correct if one can ignore any difference in the means of $V_{3}$ in the two groups.

\section{STUDIES OF PEOPLE WITH AND WITHOUT} PREVIOUS EXPOSURE

Wigaeus $e t a l^{8}$ and Löf $e t a l^{9}$ carried out a study to compare, among other things, the concentration of styrene in arterial blood between groups with and without previous styrene exposure. The unexposed group was composed of eight healthy male volunteers without a history of styrene exposure, whereas the exposed group was composed of eight workers routinely exposed to styrene. All subjects in both groups were exposed to styrene at a concentration of $80 \mathrm{ppm}$ for two hours during light physical exercise at a work load of $50 \mathrm{~W}$. The concentration of styrene in arterial blood was measured several times for each subject during and after styrene exposure. Each subject's height and weight were also measured. Table 1 shows the raw data that are used in this investigation.

\section{Results}

Table 2 shows the estimated adipose tissue volume $\hat{V}_{2}$, decay constant $\hat{\alpha}_{3}$ and clearance $\hat{\mathbf{X}}$ for each subject in the two groups. $V_{2}$ is esti- 
Table 3 Data used in the standard pharmacokinetic model

\begin{tabular}{|c|c|c|}
\hline & Rest & Workload 50W \\
\hline Total cardiac output (1/min): $\mathbf{Q}$ & $6 \cdot 0^{19}$ & $9 \cdot 9^{20}$ \\
\hline Local blood flow to tissue group 1 ( $1 / \mathrm{min}): \mathrm{Q}_{1}$ & $4 \cdot 1^{19}$ & $8 \cdot 0^{\star}$ \\
\hline Local blood flow to tissue group 2 (1/min): $\mathrm{Q}_{2}$ & 0.3219 & $0 \cdot 32^{\star}$ \\
\hline Local blood flow to tissue group $3(1 / \mathrm{min}): \mathrm{Q}_{3}$ & 1.5 & $1 \cdot 5^{\star}$ \\
\hline Volume of tissue group 1 (1): $V_{1} 319$ & $37 \cdot 3$ & $37 \cdot 3$ \\
\hline Volume of tissue group $2(1): V_{2}{ }^{19}$ & $14 \cdot 5$ & $14 \cdot 5$ \\
\hline Volume of tissue group $3(1): V_{3}{ }^{3}$ & $1 \cdot 7$ & $1 \cdot 7$ \\
\hline Tissue/blood partition coefficient for group 1: $\lambda_{1}{ }^{3}$ & $1 \cdot 2$ & $1 \cdot 2$ \\
\hline Tissue/blood partition coefficient for group $2: \lambda_{2}{ }^{3}$ & $71 \cdot 0$ & $71 \cdot 0$ \\
\hline Tissue/blood partition coefficient for group $3: \lambda_{3}{ }^{3}$ & $2 \cdot 6$ & $2 \cdot 6$ \\
\hline Air/blood partition coefficient: $\lambda_{a b}{ }^{3}$ & $1 / 59$ & $1 / 59$ \\
\hline Alveolar ventilation $(1 / \mathrm{min}): Q_{\mathrm{air}}$ & 4.915 & $20 \cdot 0^{2}$ \\
\hline
\end{tabular}

^Assume that the excess cardiac output relative to the rest state will only go to the muscle group.

mated based on a person's height $H(m)$ and weight $\mathrm{W}(\mathrm{kg})$ according to the formula:

$$
\hat{V}_{2}=1 \cdot 32\left(W-26 \cdot 69 H^{1 \cdot 424}\right)
$$

This estimator is based on a modification of Döbeln's work, ${ }^{12}$ where the product of the sums of right and left femoral condylar breadth and bistyloid radioulnar breadth were replaced by a constant; the factor 1.32 is used to convert the amount of fat $(\mathrm{kg})$ to the corresponding volume (1). The decay constant $\alpha$ for each subject is estimated by fitting equation (4) to the observed blood concentration decay curve from $\mathrm{t}=75$ to 120 minutes (three to four data points) for the treatment group and from 90 to 240 minutes (four to five data points) for the control group. The clearance is estimated with equation (8), with the partition coefficient $\lambda_{2}$ taken from table 3 .

As we are not willing to make any assumptions about underlying population distributions and as group specific sample sizes are small, we used a Wilcoxon non-parametric rank sum test to test for a significant difference in the means of the estimated clearance values in the two groups. We obtained a $P$ value of $0 \cdot 17$, implying that the difference in the estimated means of $X$ in the two groups is not significant. Note that the $P$ value for a two sample $t$ test is 0.12 (variances are not significantly different between the two groups: $\mathbf{P}=$ 0.09 ), which is in agreement with the nonparametric Wilcoxon test. In both tests, we excluded subject 3 (adipose tissue volume was not estimable from equation (12)) in the group with previous exposure, and subjects 3 (experimental data was not available to allow estimation of $\alpha$ ) and 5 (adipose tissue volume was not estimable from equation (12)) in the group without previous exposure. Therefore, the total number of subjects were seven and six in the groups with and without previous exposure to styrene, respectively.

\section{Discussion}

Filser $e t a l^{7}$ showed that linear metabolism was operative in rats and mice exposed to styrene up to $1.28 \mathrm{mg} / 1$ (300 ppm). Mendrala et $a l^{6}$ estimated the maximum rate of metabolism $\mathrm{V}_{\max }(\mathrm{mg} / \mathrm{min})$ and the Michaelis-Menten constant $K_{m}(\mathrm{mg} / \mathrm{l})$ in humans, in vitro. In that case, the estimate of $K_{m}$ was $9.4 \mathrm{mg} / 1$. Löf and Johanson $^{13}$ showed that metabolic saturation did not occur at concentrations below 250 ppm styrene $(1.06 \mathrm{mg} / \mathrm{l})$ in human volunteers. Therefore, equation (2) is appropriate for the subjects in our comparison who were exposed to $80 \mathrm{ppm}$ of styrene. However, if the exposure concentration was higher than about 250 ppm of styrene, the equation would be modified to account for saturable metabolism; that is:

$\frac{d C_{3}(t)}{d t}=\tau_{3}\left[C_{a r}(t)-\frac{C_{3}(t)}{\lambda_{3}}\right]-\frac{V_{\max } C_{3}(t)}{V_{3}\left(K_{m}+C_{3}(t)\right)}$

In this case, the linear asymptotic behaviour (3) where

$$
\kappa \cong \frac{\mathrm{V}_{\max }}{\mathrm{V}_{3} \mathrm{~K}_{\mathrm{m}}}
$$

no longer holds and equations (4)-(6) would not apply.

It is also possible to estimate $\kappa$ from normal pharmacokinetic models ${ }^{14}$ and then make a comparison of the estimated mean of $\kappa$ among subjects in the two groups to assess the appropriateness of our proposed methodology. As the partial pressure of styrene in the lung equilibrates within a fraction of a second with that in the alveolar air, the quantity of styrene entering the pulmonary compartment by inhalation and venous blood equals that leaving the compartment by exhalation and arterial blood. Therefore, we have:

$$
C_{a r t}(t)=\frac{Q_{a i r} C_{i n h}(t)+\sum_{i=1}^{3} Q_{i} \frac{C_{i}(t)}{\lambda_{i}}}{Q+Q_{a i r} \lambda_{a / b}}
$$

where $C_{\text {inh }}(\mathrm{t})$ is the concentration of styrene in the inhaled air and $Q$ is total cardiac output. The effective tissue/blood partition coefficient for each tissue group is the volume weighted average of each individual tissue partition coefficient in that group:

$$
\lambda_{i}=\frac{\sum_{j} \lambda_{i j} V_{j}}{\sum_{j} V_{j}}
$$

For instance, the lean tissue group in our model can be divided into two tissue subgroups: the blood vessel rich tissues and the skin and muscle. The volumes of blood vessel rich tissues and the skin and muscle tissues have been estimated ${ }^{3}$ to be $7 \cdot 1$ and $36 \cdot 3$ litres, respectively, while the tissue/blood partition coefficients for styrene have been estimated to be $2 \cdot 54^{3}$ and $1 \cdot 0,{ }^{15}$ respectively. Therefore, we obtain $\hat{\lambda}_{i}=1.2$ from equation (15). As most physiological variables cannot be directly measured on an individual basis, some population standard values are usually used. Table 3 lists all those literature based standard values that are needed in this model. Now, $\kappa$ can be estimated by fitting the model (equation (1), (2), and (14)) to the experimental data on styrene concentrations in arterial blood. We find many possible estimated values for $\kappa$. The range of our possible estimates of $\kappa$ could be as large as 20 -fold when allowing those standard values to vary over reasonable ranges. ${ }^{16}$ This undesirable phenomenon has been recognised by 
many others-for example, Watanabe et al. ${ }^{17}$ As with any compartmental model, the uncertainties associated with these standard values will necessarily lead to uncertainties about the key variables. Therefore, valid statistical comparisons cannot be made based on these estimated values of $\kappa$

The error involved in estimating $\alpha$ from our data might be large because the observations were obtained in only two to four hours after the end of exposure. Ramsey et al ${ }^{18}$ monitored the decay behaviour of styrene in blood of four male volunteers over 47 hours after exposure to $82 \mathrm{ppm}$ styrene for six hours. The half life for the terminal decay phase was measured to be $13.0( \pm 0.7)$ hours, which translates into an $\alpha$ value of $0.053( \pm 0.003) \mathrm{h}^{-1}$. These values are about $1 / 12$ and $1 / 6$ of those we estimated for the groups with and without previous exposure to styrene, respectively. Therefore, the decay constants of Wigaeus et $a l^{8}$ and Löf et $a l^{9}$ may indeed be too large to represent the real terminal decay behaviour.

Strictly speaking, equation (8) cannot be used if $\alpha$ is derived from the decay behaviour well before equilibrium has been established throughout the body, including the fat tissues. This condition is rarely met rigorously in practice and may constitute the limitation of the application of this method. However, in the event that this equilibrium was not achieved in this study, we think that our conclusion that styrene exposure did not increase metabolic activity would still be valid as the shorter period of observation for the subjects with previous exposure relative to those without $(2 \mathrm{~h} v$ $4 \mathrm{~h}$ ) would have exaggerated the differences in $\kappa$ values between the two groups (equation (11)).

This result disagrees with that of Löf et al. ${ }^{9}$ We think that the main reason for this discrepancy is the higher volume of distribution of styrene in the pre-exposed subjects due to their larger amounts of adipose tissue. As we stated, the decay constants estimated by Wigaeus $e t a l^{8}$ and Löf $e t a l^{9}$ were likely to be more overestimated for the pre-exposed group than for the group without previous exposure. As a result, the estimated areas under the blood concentration $v$ time curves for the two groups would tend to be significantly different. However, as the data set is small and as the data on styrene concentration do not exactly reflect the true asymptotic decay behaviour, we think that further studies are needed to draw more definitive conclusions.

Finally, we will make a few comments about our estimation of $\kappa$. Taking $\alpha=0.053 \mathrm{~h}^{-1}$ by Ramsey $e t a l^{18}$ and other data from table 3, we find from equation (8) and (11) that $\kappa=0 \cdot 2 / \mathrm{min}$. Mendrala et $\mathrm{al}^{6}$ measured the Michaelis-Menten constants $V_{\max }$ and $K_{m}$ associated with styrene with human livers in vitro to be $3.2 \mathrm{mg} / \mathrm{h} / \mathrm{kg}$ body weight and
$0.09 \mathrm{mmol} / 1$, respectively. From equation (13), this translates into a $\kappa$ value of about $0 \cdot 2 / \mathrm{min}$ when assuming $70 \mathrm{~kg}$ body weight and a liver volume of 1.71 (see table 3 ) for a standard man. Therefore, our estimate agrees very well with the experimental results obtained from human livers in vitro. In contrast, $\kappa$ would be $2 \cdot 57 / \mathrm{min}$ by scaling up the variables for rats, ${ }^{15}$ and $\kappa$ could vary anywhere from $0 \cdot 1 / \mathrm{min}$ to $3.0 / \mathrm{min}$ with least squares methods when allowing $30 \%$ variation for the standard physiological variables. This again points to the desirability of estimating human kinetic variables from human data rather than from extrapolation based on animal experimental data.

1 Barale $R$. The genetic toxicology of styrene and styrene oxide. Mutat Res 1991;257:107-26.

2 Leibman KC, Ortiz E. Epoxide intermediates in microsomal oxidation of olefins to glycols. F Pharmacol Exp Ther 1970;173:242-6.

3 Droz PO, Guillemin MP. Human styrene exposure. V: development of a model for biological monitoring. Int Arch Occup Environ Health 1983;53:19-36.

4 Andersen ME, Gargas ML, Ramsey JC. Inhalation pharmacokinetics: evaluating systemic extraction, total in vivo metabolism, and the time course of enzyme induction fo inhaled styrene in rats based on arterial blood: inhaled air concentration ratios. Toxicol Appl Pharmacol 1984;73: 176-87.

5 Elovaara E, Engström K, Nakajima T, Park SS, Gelboin HV, Vainio H. Metabolism of inhaled styrene in acetone, phenobarbital and 3-methylcholanthrene-pretreated rats: stimulation and stereochemical effects by induction of cytochromes P450IIE1, P450IIB and P450IA. Xenobiotica 1991;21:651-61.

6 Mendrala AL, Langvardt PW, Nitschke KD, Quast JF, Nolan RJ. In vitro kinetics of styrene and styrene oxide Nolan RJ. In vitro kinetics of styrene and styrene oxide metabolism in

7 Filser JG, Schwegler U, Csanady GA, Greim H, Kreuzer PE, Kessler W. Species-specific pharmacokinetics of styrene in rat and mouse. Arch Toxicol 1993;67:517-30.

8 Wigaeus E, Löf A, Bjurström R, Nordqvist MB. Exposure to styrene. Scand $\mathcal{f}$ Work Environ Health 1983;9:479-88.

9 Löf A, Lundgren E, Nordqvist MB. Kinetics of styrene in workers from a plastics industry after controlled exposure: a comparison with subjects not previously exposed. Br f Ind Med 1986;43:537-43

10 Nakajima T, Elovaara E, Gonzalez FJ, Gelboin HV, Raunio H, Pelkonen O, et al. Styrene metabolism by cDNA-expressed human hepatic and pulmonary cytocDNA-expressed human hepatic and pulmonary

11 Sato A, Nakajima T, Fujiwara Y, Hirosawa K. Pharmacokinetics of benzene and toluene. Int Arch Arheitsmed 1974;33:169-82.

12 von Döbeln $W$. Anthropometric determination of fat-free body weight. Acta Med Scand 1959;165:37-40.

13 Löf A, Johanson G. Dose-dependent kinetics of inhaled styrene in man. In: Sorsa M, Peltonen $\mathrm{K}$, Vainio $\mathrm{H}$ Hemminki K, eds. LARC Sci Publ 1993;127:89-99.

14 Gibaldi M, Perrier D. Pharmacokinetics (2nd ed). New York, NY: Marcel Dekker, 1982

15 Ramsey JC, Andersen ME. A physiologically based description of the inhalation pharmacokinetics of styrene in rats and humans. Toxicol Appl Pharmacol 1984;73:159-75.

16 Droz PO. Quantification of biological variability. Ann Occup Hyg 1992;36:295-306.

17 Watanabe KH, Bois FY, Daisey JM, Auslander DM, Spear RC. Benzene toxicokinetics in humans: exposure of bone marrow to metabolites. Occup Environ Med 1994;51: marrow

18 Ramsey JC, Young JD, Karbowski RJ, Chenoweth MB, McCarty LP. Pharmacokinetics of inhaled styrene in human volunteers. Toxicol Appl Pharmacol 1980;53 54-63.

19 Saidman LJ. Anesthesia at a constant alveolar concentration. In: Fiserova-Bergerova V, ed. Modeling of inhalation exposure to vapors: uptake, distribution, and elimination. Vol II. Florida: CRC Press, 1983:131-43.

20 Astrand I. Effect of physical exercise on uptake, distribution, and elimination of vapors in man. In: FiserovaBergerova V, ed. Modeling of inhalation exposure to vapors: uptake, distribution, and elimination. Vol II. Florida: CRC Press, 1983:107-30. 